\title{
The Effect of Interferometer Phase Error on Direct-Detection DPSK and DQPSK Signals
}

\author{
Keang-Po Ho, Member, IEEE
}

\begin{abstract}
Based on the well-known Marcum's $Q$ function, closed-form formulae are derived to evaluate the error probability of direct-detected differential phase-shift keying (DPSK) and differential quadrature phase-shift keying (DQPSK) signals with interferometer phase error. For a signal-to-noise ratio penalty less than $1 \mathrm{~dB}$, the phase error must be less than $16^{\circ}$ and $6^{\circ}$ for DPSK and DQPSK signals, respectively.
\end{abstract}

Index Terms-Differential phase-shift keying (DPSK), differential quadrature phase-shift keying (DQPSK), direct detection, phase error.

\section{INTRODUCTION}

D IFFERENTIAL phase-shift keying (DPSK) [1]-[5] and differential quadrature phase-shift keying (DQPSK) [6]-[9] modulation have received renewed attention recently for long-haul transmission or spectrally efficiency systems. When DPSK and DQPSK signals are directly detected using a balanced receiver following an interferometer, one of the major receiver imperfections is the phase error at the asymmetric Mach-Zehnder interferometer [10]-[14]. Although the phase error can be eliminated by active delay-line stabilization [10], the degradation due to phase error needs to be calculated accurately, as an application, to specify the accuracy requirement for the stabilization algorithm.

Previous works evalulate the sensitivity penalty due to phase error by experiment [12], simulation [11], [14], or semianalytical technique [13]. As shown in the Appendix , for a matched filter-based receiver, the formula from [15] can be used to calculate the symbol or bit-error rate (BER) with phase error. However, the series summation from [15] has large terms oscillating between positive and negative values, giving some numerical difficulties. The closed-form expression of [15] is not used in [11], [12], [14].

In this letter, analytical expressions based on the well-known Marcum's $Q$ function [16, pp. 43-44] are used to evaluate the error probability for DPSK and DQPSK signals with phase error. Originally for noncoherent detection of correlated binary signals, the formulae from [16, Sec. 5.4.4] are adapted for DPSK and DQPSK signals with phase error. The correlation coefficient, an essential parameter, as a function of phase error is given for the first time in this letter for DPSK or DQPSK signals with phase error.

Manuscript received April 25, 2003; revised July 28, 2003. This work was supported in part by the National Science Council of R.O.C. under Grant NSC-92-2218-E-002-034.

The author is with the Institute of Communication Engineering and Department of Electrical Engineering, National Taiwan University, Taipei 106, Taiwan, R.O.C. (e-mail: kpho@cc.ee.ntu.edu.tw).

Digital Object Identifier 10.1109/LPT.2003.819359

\section{DPSK SIGNALS}

If the path difference in the asymmetric interferometer does not match to the frequency of the signal, the phase error is equal to $\theta_{e}=2 \pi \Delta f T$, where $\Delta f$ is the frequency mismatch and $T$ is the symbol period. At the output of the balanced receiver, ignoring the constant factor of coupler loss, photodetector responsivity, and receiver gain, the signal is

$$
s(t)=\left|E(t)+e^{j \theta_{e}} E(t-T)\right|^{2}-\left|E(t)-e^{j \theta_{e}} E(t-T)\right|^{2} .
$$

As the signal of (1) is the same as that of the decision variable for envelope detection of correlated binary signals [16, Sec. 5.4.4], the equivalent correlation coefficient is [16, eq. (5.4-54)], denoted as $\rho$

$$
\frac{1}{2}\left|1+e^{j \theta_{e}}\right|\left|1-e^{-j \theta_{e}}\right|=\sin \theta_{e}
$$

Using [16, eq. (5.4-54)], the BER is

$$
\begin{aligned}
p_{e}= & Q_{1}(a, b)-\frac{1}{2} e^{-\left(a^{2}+b^{2}\right) / 2} I_{0}(a b), \\
& a=\sqrt{\rho_{s}\left(1-\cos \theta_{e}\right)}, \quad b=\sqrt{\rho_{s}\left(1+\cos \theta_{e}\right)}
\end{aligned}
$$

where $Q_{1}(\cdot, \cdot)$ is the well-known first-order Marcum's $Q$ function, and $\rho_{s}$ is the signal-to-noise ratio (SNR). The error probability of (3) assumes a matched filter [16, Sec. 5.1.2], the typical cases of [13], [14]. Within the matched filter, only the amplifier noise in the same polarization as the signal is considered, the typical cases of [17]-[19]. The results are applicable to both return-to-zero or nonreturn-to-zero line codes.

The BER of (3) used an SNR twice that in [16, eq. (5.4-54)] as the correlated binary signal is $E(t) \pm e^{j \theta_{e}} E(t-T)$, uses two time slots, and doubles the energy per symbol. While difficult to prove analytically, from numerical results, the BER of (3) and (5) from the Appendix are the same.

\section{DQPSK SIGNALS}

DQPSK signals have four constellation points, corresponding to four correlated signals with noncoherent envelope detection. Without phase error, the BER with Gray code is that of (3) with $a=\rho_{s}^{1 / 2}(1-1 / \sqrt{2})^{1 / 2}, b=\rho_{s}^{1 / 2}(1+1 / \sqrt{2})^{1 / 2}$ for a correlated angle of $\pi / 4[16$, eq. (5.2-71)] .

Without going into details, with phase error, the BER is

$$
\begin{aligned}
p_{e}=\frac{1}{2}\left\{Q_{1}\left(a_{+}, b_{+}\right)-\frac{1}{2} e^{-\left(a_{+}^{2}+b_{+}^{2}\right) / 2} I_{0}\left(a_{+} b_{+}\right)\right. \\
\left.+Q_{1}\left(a_{-}, b_{-}\right)-\frac{1}{2} e^{-\left(a_{-}^{2}+b_{-}^{2}\right) / 2} I_{0}\left(a_{-} b_{-}\right)\right\}
\end{aligned}
$$



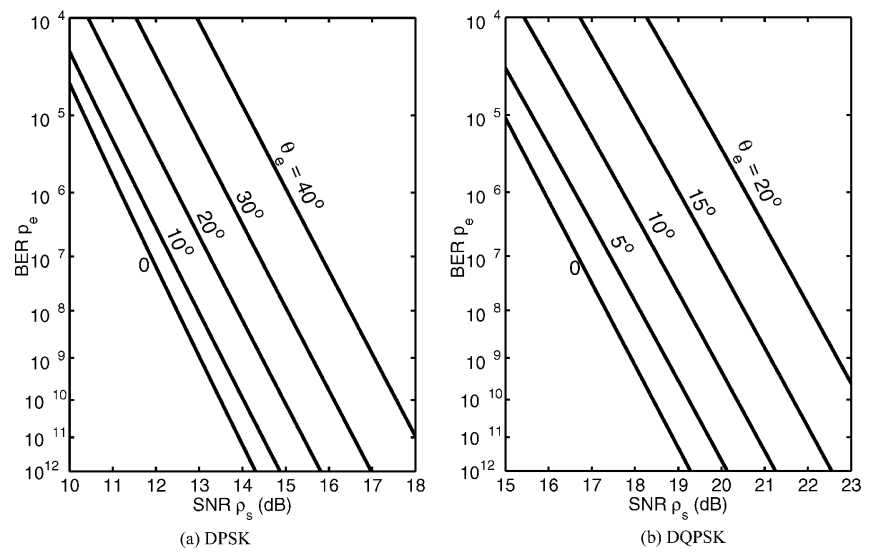

Fig. 1. BER as a function of SNR $\rho_{s}$ for (a) DPSK and (b) DQPSK signals with interferometer phase error.

$$
\begin{aligned}
& a_{ \pm}=\sqrt{\rho_{s}\left[1-\cos \left(\frac{\pi}{4} \pm \theta_{e}\right)\right]} \\
& b_{ \pm}=\sqrt{\rho_{s}\left[1+\cos \left(\frac{\pi}{4} \pm \theta_{e}\right)\right]} .
\end{aligned}
$$

The BER of (4) is numerically the same as (6) from the Appendix . From the two terms of (4), due to the phase error, the two adjacent points of the signal constellation of the DQPSK signal have different correlation coefficients. The correlated angles to the adjacent points are increased (corresponding to $a_{+}$ and $b_{+}$) or decreased (corresponding to $a_{-}$and $b_{-}$) by the phase error of $\theta_{e}$ from $\pi / 4$.

\section{NUMERICAL RESULTS}

From (3) and (4), the BER is independent of the sign of the phase error. In later parts of this letter, only the results of positive phase error are shown.

Fig. 1 shows the BER as a function of SNR $\rho_{s}$. Fig. 1(a) is plotted for a DPSK signal with phase errors of $10^{\circ}, 20^{\circ}$, $30^{\circ}$, and $40^{\circ}$. Fig. 1(a) also shows the error probability with no phase error of $p_{e}=1 / 2 \exp \left(-\rho_{s}\right)$ [16, eq. (5.2-69)]. Fig. 1(b) is plotted for a DQPSK signal with phase errors of $5^{\circ}, 10^{\circ}, 15^{\circ}$, and $20^{\circ}$. In Fig. 1(b), the BER is plotted versus the SNR instead of SNR per bit in [16, Fig. 5.2-13]. Fig. 1(b) also shows the error probability with no phase error [16, eq. (5.2-71)].

Fig. 2 shows the SNR penalty as a function of phase error for both DPSK and DQPSK signals. The SNR penalty is calculated for a BER of $10^{-9}$, corresponding to a required SNR of 13 and $18 \mathrm{~dB}$ for DPSK and DQPSK signals, respectively.

The curve of SNR penalty of a DPSK signal in Fig. 2 has insignificant difference with the corresponding curves in [12, Fig. 3], [13, Fig. 2], and [14, Fig. 5] (required the adjusting of $x$ axis). The phase error for an SNR penalty of $1 \mathrm{~dB}$ is about $16^{\circ}$ (or $4.5 \%$ of $360^{\circ}$ ) for a DPSK signal. In all of [12]-[14], the phase error of 1-dB SNR penalty is about $4 \%$ to $5 \%$ from simulation or analysis. With narrow bandwidth and from [13], the SNR penalty due to phase error is more or less independent of the optical filter before the interferometer. For SNR penalty less than $2 \mathrm{~dB}$ and from [14], the SNR penalty due to phase error is more or less independent of the electrical filtering after the balanced receiver. Just as with the theoretical results from

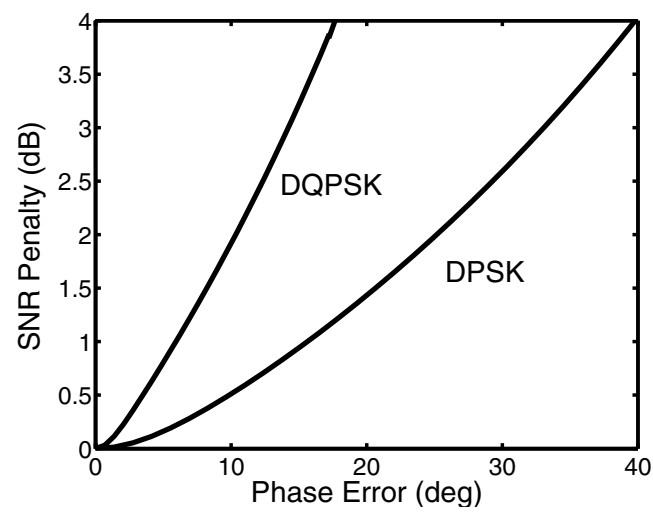

Fig. 2. SNR penalty as a function of interferometer phase error for DPSK and DQPSK signals.

[12]-[14], the SNR penalty of Fig. 2 is smaller than that from measurement [12], [14]. As explained in [14], this discrepancy is probably due to the nonideal signal source used in the experiment. The $10 \%$ (or $36^{\circ}$ ) mismatch of [11] gives a penalty of about $3.5 \mathrm{~dB}$.

For the same SNR penalty, the DQPSK signal is about 2.7 times more sensitive to the phase error than is the DPSK signal, more or less the same ratio as the experimental and simulated results in [12]. The phase error for an SNR penalty of $1 \mathrm{~dB}$ is about $6^{\circ}$ (or $1.7 \%$ of $360^{\circ}$ ) for a DQPSK signal, the same as a ratio of mismatched frequency to the symbol rate of [12, Fig. 3] by simulation. The measurement of [12] shows a larger penalty than that of Fig. 2 due to nonideal signal source [14].

\section{CONCLUSION}

Based on the well-known Marcum's $Q$ function, two analytical formulae are derived to evaluate the BER of DPSK and DQPSK signals with interferometer phase error at the receiver. The error probability is numerically verified as the same as that from the series summation of [15] and the Appendix. For an SNR penalty less than $1 \mathrm{~dB}$, the phase error of the interferometer must be less than $16^{\circ}$ and $6^{\circ}$ for DPSK and DQPSK signals, respectively, the same as the simulation or analytical results from [12]-[14].

\section{APPENDIX}

From [15], the error probability for a DPSK signal with phase error is

$$
\begin{aligned}
p_{e}=\frac{1}{2}-\frac{\rho_{s} e^{-\rho_{s}}}{2} \sum_{k=0}^{\infty} \frac{(-1)^{k}}{2 k+1}\left[I_{k}\left(\frac{\rho_{s}}{2}\right)\right. & \left.+I_{k+1}\left(\frac{\rho_{s}}{2}\right)\right]^{2} \\
& \times \cos \left[(2 k+1) \theta_{e}\right]
\end{aligned}
$$

where $I_{k}(\cdot)$ is the $k$ th order modified Bessel function of the first kind.

From the factor of $(-1)^{k}$, the terms of (5) oscillate between positive and negative values. Although the summation of (5) converges, the calculation is numerically challenging for small error probability. Note that the multiplication factor of the summation is a small value of $\rho_{s} e^{-\rho_{s}} / 2$, and the summation has a value of about $e^{\rho_{s} / 2} / \rho_{s}$ for small error probability. For large $\operatorname{SNR} \rho_{s}$, the summation has very large terms although the error 
probability is small. The error probability is the difference between $1 / 2$ and a value a little bit smaller than $1 / 2$. As a comparison, the Marcum's $Q$ function is the summation of all positive small terms [16, pp. 43-44].

For DQPSK signals, following the derivation of [15], we get the BER as

$$
\begin{aligned}
p_{e}=\frac{3}{8}- & \frac{\rho_{s} e^{-\rho_{s}}}{4} \sum_{m=1}^{\infty} \frac{\sin \left(\frac{m \pi}{4}\right)}{m} \\
& \times\left[I_{m-1 / 2}\left(\frac{\rho_{s}}{2}\right)+I_{m+1 / 2}\left(\frac{\rho_{s}}{2}\right)\right]^{2} \cos \left(m \theta_{e}\right) .
\end{aligned}
$$

Similar to (5), the summation in (6) is difficult to calculate for small BER. Although the BER of (6) has never been derived before, it comes almost directly from [15].

\section{REFERENCES}

[1] A. H. Gnauck, G. Raybon, S. Chandrasekhar, J. Leuthold, C. Doerr, L. Stulz, A. Agrawal, S. Banerjee, D. Grosz, S. Hunsche, A. Kung, A. Marhelyuk, D. Maymar, M. Movassaghi, X. Liu, C. Xu, X. Wei, and D. M. Gill, " $2.5 \mathrm{~Tb} / \mathrm{s}(64 \times 42.7 \mathrm{~Gb} / \mathrm{s})$ transmission over $40 \times 100$ km NZDSF using RZ-DPSK format and all-raman-amplified spans," in Tech. Dig. Optical Fiber Communications Conf. 2002, Anaheim, CA, Postdeadline Paper FC2.

[2] A. H. Gnauck, G. Raybon, S. Chandrasekhar, J. Leuthold, C. Doerr, L. Stulz, and E. Burrows, " 25 40-Gb/s copolarized DPSK transmission over 12 100-km NZDF with 50-GHz channel spacing," IEEE Photon. Technol. Lett., vol. 15, pp. 467-469, Mar. 2003.

[3] C. Rasmussen, T. Fjelde, J. Bennike, F. Liu, S. Dey, B. Mikkelsen, P. Mamyshev, P. Serbe, P. van de Wagt, Y. Akasaka, D. Harris, D. Gapontsev, V. Ivshin, and P. Reeves-Hall, "DWDM 40 G transmission over trans-pacific distance $(10,000 \mathrm{~km})$ using CSRZ-DPSK, enhanced FEC and all-raman amplified $100 \mathrm{~km}$ Ultra-Wave fiber spans," in Tech. Dig. Optical Fiber Communications Conf. 2003, Atlanta, GA, Postdeadline Paper PD18.

[4] B. Zhu, L. E. Nelson, S. Stulz, A. H. Gnauck, C. Doerr, J. Leuthold, L. Grüner-Nielsen, M. O. Pederson, J. Kim, R. Lingle, Y. Emori, Y. Ohki, N. Tsukiji, A. Oguri, and S. Namiki, "6.4-Tb/s $(160 \times 42.7 \mathrm{~Gb} / \mathrm{s})$ transmission with $0.8 \mathrm{bit} / \mathrm{s} / \mathrm{Hz}$ spectral efficiency over $32 \times 100 \mathrm{~km}$ of fiber using CSRZ-DPSK format," in Tech. Dig. Optical Fiber Communications Conf. 2003, Atlanta, GA, Postdeadline Paper PD19.
[5] C. Xu, X. Liu, L. F. Mollenauer, and X. Wei, "Comparison of return-to-zero differential phase-shift keying and on-off keying long-haul dispersion managed transmission," IEEE Photon. Technol. Lett., vol. 15, pp. 617-619, Apr. 2003

[6] R. A. Griffin, R. I. Johnstone, R. G. Walker, J. Hall, S. D. Wadsworth, K. Berry, A. C. Carter, M. J. Wale, P. A. Jerram, and N. J. Parsons, "10 $\mathrm{Gb} / \mathrm{s}$ optical differential quadrature phase shift key (DQPSK) transmission using GaAs/AlGaAs integration," in Tech. Dig. Optical Fiber Communications Conf. 2002, Anaheim, CA, Postdeadline Paper FD6.

[7] P. S. Cho, V. S. Grigoryan, Y. A. Godin, A. Salamon, and Y. Achiam, "Transmission of 25-Gb/s RZ-DQPSK signals with $25-\mathrm{GHz}$ channel spacing over $1000 \mathrm{~km}$ of SMF-28 fiber," IEEE Photon. Technol. Lett., vol. 15 , pp. 473-475, Mar. 2003

[8] C. Wree, N. Hecker-Denschlag, E. Gottwald, P. Krummrich, J. Leibrich, E.-D. Schmidt, B. Lankl, and W. Rosenkrantz, "High spectral efficiency $1.6-\mathrm{b} / \mathrm{s} / \mathrm{Hz}$ transmission $(8 \times 40 \mathrm{~Gb} / \mathrm{s}$ with a $25-\mathrm{GHz}$ grid) over $200-\mathrm{km}$ SSMF using RZ-DQPSK and polarization multiplexing," IEEE Photon. Technol. Lett., vol. 15, pp. 1303-1305, Sept. 2003.

[9] R. Griffin, R. Johnstone, R. Walker, S. Wadsworth, A. Carter, and M. Wale, "Integrated DQPSK transmitter for dispersion-tolerant and dispersion-managed DWDM transmission," in Tech. Dig. Optical Fiber Communications Conf. 2002, Atlanta, GA, Paper FP6.

[10] E. A. Swanson, J. C. Livas, and R. S. Bondurant, "High sensitivity optically preamplified direct detection DPSK receiver with active delay-line stabilization," IEEE Photon. Technol. Lett., vol. 6, pp. 263-265, Feb. 1994.

[11] M. Rohde, C. Caspar, N. Heimes, M. Konitzer, E.-J. Bachus, and N. Hanik, "Robustness of DPSK direct detection transmission format in standard fiber WDM systems," Electron. Lett., vol. 36, no. 17, pp. 1483-1484, 2000.

[12] H. Kim and P. Winzer, "Robustness to laser frequency offset in directdectection DPSK and DQPSK systems," J. Lightwave Technol., vol. 21, pp. 1887-1891, Sept. 2003.

[13] G. Bosco and P. Poggiolini, "The effect of receiver imperfections on the performance of direct-detection optical systems using DPSK modulation," in Tech. Dig. Optical Fiber Communications Conf. 2002, Atlanta, GA, Paper ThE6.

[14] P. J. Winzer and H. Kim, "Degraduations in balanced DPSK receivers," IEEE Photon. Technol. Lett., vol. 15, pp. 1282-1284, Sept. 2003.

[15] N. M. Blachman, "The effect of phase error on DPSK error probability," IEEE Trans. Commnun., vol. COM-29, pp. 364-465, Mar. 1981.

[16] J. G. Proakis, Digital Communications, 4th ed. New York: McGrawHill, 2000.

[17] P. A. Humblet and M. Azizoglu, "On the bit error rate of lightwave systems with optical amplifiers," J. Lightwave Technol., vol. 9, pp. 1576-1582, Nov. 1991.

[18] J. J. O. Pires and J. R. F. de Rocha, "Performance analysis of DPSK direct detection optical systems in the presence of interferometric intensity," $J$. Lightwave Technol., vol. 10, pp. 1722-1730, Nov. 1992.

[19] S. R. Chinn, D. M. Boroson, and J. C. Livas, "Sensitivity of optically preamplified DPSK receivers with Fabry-Pérot filters," J. Lightwave Technol., vol. 14, pp. 370-376, Mar. 1996. 